\title{
Inhibidores de escaso impacto medioambiental. Sistemas basados en tierras raras $^{\left({ }^{\circ}\right.}$
}

\author{
A. Aballe $\left(^{(*)}\right.$, M. Bethencourt $\left(^{(*)}\right.$, F.J. Botana ${ }^{(*)}$, M. Marcos ${ }^{(* *)}$, J. Pérez ${ }^{(*)}$ \\ y M.A. Rodríguez $\left.{ }^{*}\right)$
}

Resumen Se estudia el comportamiento de los cloruros de lantano, cerio y samario como inhibidores de los procesos de corrosión uniforme y por picaduras de los aceros inoxidables AISI 434 y AISI 304 y de la aleación de aluminio AA 5083 en disoluciones aireadas de $\mathrm{NaCl}$ al 3,5\%. Utilizando técnicas electroquímicas como polarización lineal y cíclica, se ha podido evaluar el poder inhibidor de estas sustancias. El mejor comportamiento inhibidor se ha obtenido para una concentración de $500 \mathrm{ppm} \mathrm{de}$ $\mathrm{CeCl}_{3}$, si bien para los aceros se obtienen resultados del mismo orden para $500 \mathrm{ppm} \mathrm{de} \mathrm{LaCl}_{3}$. Con la ayuda de microscopía electrónica de barrido y espectroscopía de dispersión de energía se ha podido confirmar el carácter catódico del mecanismo de inhibición que tiene lugar en el proceso.

Palabras clave: Tierras raras. Inhibidores ecológicos. Corrosión uniforme. Corrosión por picaduras. Agua de mar.

\section{Green inhibitors. Rare Earth based systems}

\begin{abstract}
Lanthanum, Cerium and Samarium chlorides have been investigated as uniform and pitting corrosion inhibitors of AISI 434 and AISI 304 stainless steels and AA $5083 \mathrm{Al}-\mathrm{Mg}$ alloy in $3.5 \% \mathrm{NaCl}$ aerated aqueous solutions. Their inhibitor power was evaluated by using electrochemical techniques such as Linear and Cyclic Polarisation. In each case, the highest protection degree was found in the solution dopped with $500 \mathrm{ppm}$ of $\mathrm{CeCl}_{3}$. Similar results were obtained for additions of $500 \mathrm{ppm}$ of $\mathrm{LaCl}_{3}$. Scanning Electron Microscopy and Energy Dispersive Spectroscopy allowed us to confirm the cathodic nature of the inhibition process.
\end{abstract}

Keywords: Rare Earths. Green inhibitors. Uniform corrosion. Pitting. Seawater.

\section{INTRODUCCIÓN}

En la actualidad, se utiliza una amplia gama de compuestos inorgánicos como inhibidores de la corrosión metálica en sistemas acuosos (1). De ellos, uno de los sistemas más usados, por su versatilidad y su excelente relación efectividad-coste, es el basado en compuestos de $\mathrm{Cr}(\mathrm{VI})$, fundamentalmente en forma de cromatos (2). Sin embargo, en los últimos años ha quedado suficientemente demostrado el peligro que supone el uso de estos compuestos, tanto por su elevada toxicidad (3)

(•) Trabajo recibido el día 24 de enero de 1997.

(*) Dpto. de Ciencia de los Materiales e Ingeniería Metalúrgica y Química Inorgánica. Facultad de Ciencias. Universidad de Cádiz. Apdo 40. 11510-Puerto Real. Cádiz (España).

(**) Dpto. de Ingeniería Mecánica y Diseño Industrial. Escuela Superior de Ingeniería. Universidad de Cádiz. Sacramento, 82. 11003-Cádiz (España). como por su negativa repercusión en el medio ambiente (4). Esto ha llevado a diferentes investigadores a dirigir sus esfuerzos hacia la búsqueda de alternativas eficaces de escaso impacto medioambiental al uso de estos compuestos. En esa línea se ha estudiado el comportamiento como inhibidores de diversos compuestos orgánicos, tales como benzoatos y glucosatos, e inorgánicos, como fosfatos, molibdatos, silicatos, nitritos o sales de cinc (5). Asimismo, para generar capas libres de cromo se vienen estudiando métodos diversos como el anodizado sulfúrico, con el que se consigue depositar sobre el metal películas finas de elevada resistencia a la corrosión (6), la formación pirolítica de capas de óxido de estaño (7), el desarrollo de películas cerámicas por chispa anódica (8), la deposición de capas por el método sol-gel (9) o la incorporación de elementos lantánidos (10-18). En nuestros laboratorios se viene estudiando desde hace algunos años el comportamiento de sales lantánidas (19-24) como inhibidores de la corrosión, dado el bajo nivel 
de toxicidad presentado por las mismas (25) y los resultados previos obtenidos por otros autores.

En el presente trabajo se analiza el efecto producido por la adición de sales lantánidas del tipo $\mathrm{LnCl}_{3}$ sobre la corrosión generalizada y por picaduras en dos tipos de acero, AISI 434 y 304, y en una aleación de Al-Mg, AA 5083, en disoluciones aireadas de $\mathrm{NaCl}$.

\section{PARTE EXPERIMENTAL}

En los ensayos se han utilizado probetas de acero AISI 434 y 304 de $30 \times 25 \times 1,5 \mathrm{~mm}$, cuya composición muestra la tabla 1 , y de la aleación de aluminio AA 5083 de $30 \times 25 \times 5 \mathrm{~mm}$, cuya composición queda recogida en la tabla II.

Antes de los ensayos, las probetas se pulieron a 220 y 500 grits. Las probetas de acero se desengrasaron con benceno en ebullición, mientras que las de la aleación de aluminio lo fueron con hexano. Posteriormente, los tres tipos se lavaron con agua destilada. A las probetas destinadas a los ensayos de inmersión se les practicó un orificio de aproximadamente $3 \mathrm{~mm}$ de diámetro en la cercanía de una de las aristas de $25 \mathrm{~mm}$, centrado respecto a la misma, con objeto de ubicar la probeta en la celda de ensayo.

Los ensayos se llevaron a cabo a temperatura ambiente, en disolución de $\mathrm{NaCl}$ al 3,5\% en peso saturada de oxígeno. El efecto inhibidor de las sales lantánidas se evaluó añadiendo a las disoluciones cantidades variables, entre 100 y 1.000 ppm, de $\mathrm{LaCl}_{3}, \mathrm{CeCl}_{3}$ y $\mathrm{SmCl}_{3}$.

En los ensayos de inmersión se utilizó una celda diseñada siguiendo las especificaciones de la norma ASTM G-31. Para evaluar la pérdida de peso de las muestras sometidas a este tipo de ensayo, se eliminaron los productos de corrosión adheridos a las mismas tal y como especifica la norma ASTM G-1. Antes de este último proceso, las superficies de las muestras se observaron con un microscopio electrónico de barrido (MEB) JEOL 820-SM. Dichas muestras también se estudiaron mediante espectroscopía de dispersión de energía (EDS) con un analizador AN-10000, de la marca Link, acoplado al citado microscopio.

Las medidas electroquímicas de polarización lineal y de polarización cíclica se llevaron a cabo
TABLA II.- Composición de la aleación AA5083, $\%$ en peso

TABLE II.- AA5083 weight percent composition

\begin{tabular}{|ccccccc|}
\hline $\mathrm{Mg}$ & $\mathrm{Mn}$ & $\mathrm{Si}$ & $\mathrm{Fe}$ & $\mathrm{Ti}$ & $\mathrm{Cu}$ & $\mathrm{Cr}$ \\
\hline 4,50 & 0,70 & 0,55 & 0,40 & 0,15 & 0,10 & $<0,25$ \\
\hline
\end{tabular}

empleando indistintamente un potenciostato Parc, modelo VersaStat, y otro Solartron, modelo 1287, controlados desde un ordenador compatible PC mediante los programas M352, de Parc, y CorrWare para Windows, de Solartron. Dichos ensayos se llevaron a cabo en una celda plana K235 Flat Cell, de Parc, y en una celda construida según la norma ASTM G-5, utilizando como electrodos de referencia, respectivamente, uno de $\mathrm{Ag} / \mathrm{AgCl}$ saturado en $\mathrm{KCl}$ y otro de calomelanos. Los valores de potencial que se ofrecen en el presente trabajo se refieren al electrodo de $\mathrm{Ag} / \mathrm{AgCl}$. Para ambos tipos de ensayos se ha empleado una velocidad de polarización de $10 \mathrm{mV} \cdot \mathrm{min}^{-1}$. Para los ensayos de polarización lineal, se tomó un intervalo de tensión que evitara la aparición de fenómenos de corrosión localizada. Por otro lado, para los ensayos de polarización cíclica, se tomó como punto de retorno el correspondiente a un potencial de $5 \mathrm{mV}$ por encima del de corrosión, con una densidad de corriente anódica de $0,5 \mathrm{~mA} \cdot \mathrm{cm}^{-2}$ que garantiza la formación de picaduras.

\section{RESULTADOS Y DISCUSIÓN}

La figura 1 muestra la evolución con el tiempo de exposición de la pérdida de peso sufrida por la aleación de aluminio, sometida a ensayos de inmersión total en la disolución de $\mathrm{NaCl}$ y con adición de 500 ppm de cada una de las sales empleadas. Se puede observar cómo en el intervalo de tiempo estudiado la adición de las sales del tipo $\mathrm{LnCl}_{3}$ provoca un descenso de la pérdida de peso de la aleación con respecto a la obtenida en la disolución de $\mathrm{NaCl}$. Dada la relación directa existente entre la pérdida de peso y la velocidad de corrosión uniforme, estos primeros resultados indican que la presencia de sal lantánida en la disolución inhibe este tipo de corrosión. Por otro lado, en la misma figura es posible observar cómo a medida que se avanza

TABLA I.- Composición de los aceros AISI 304 y AISI 434, \% en peso

TABLE I.- AISI 434 and AISI 304 SS weight percent composition

\begin{tabular}{|c|ccccccccc|}
\hline & $\mathrm{C}$ & $\mathrm{Si}$ & $\mathrm{Mn}$ & $\mathrm{Cr}$ & $\mathrm{Ni}$ & $\mathrm{Mo}$ & $\mathrm{P}$ & $\mathrm{N}$ & $\mathrm{Cu}$ \\
\hline AISI 304 & 0,048 & 0,35 & 1,37 & 18,30 & 8,11 & 0,26 & 0,028 & 0,049 & 0,18 \\
AISI 434 & 0,023 & 0,39 & 0,33 & 16,28 & 0,16 & 0,95 & 0,010 & - & 0,10 \\
\hline
\end{tabular}




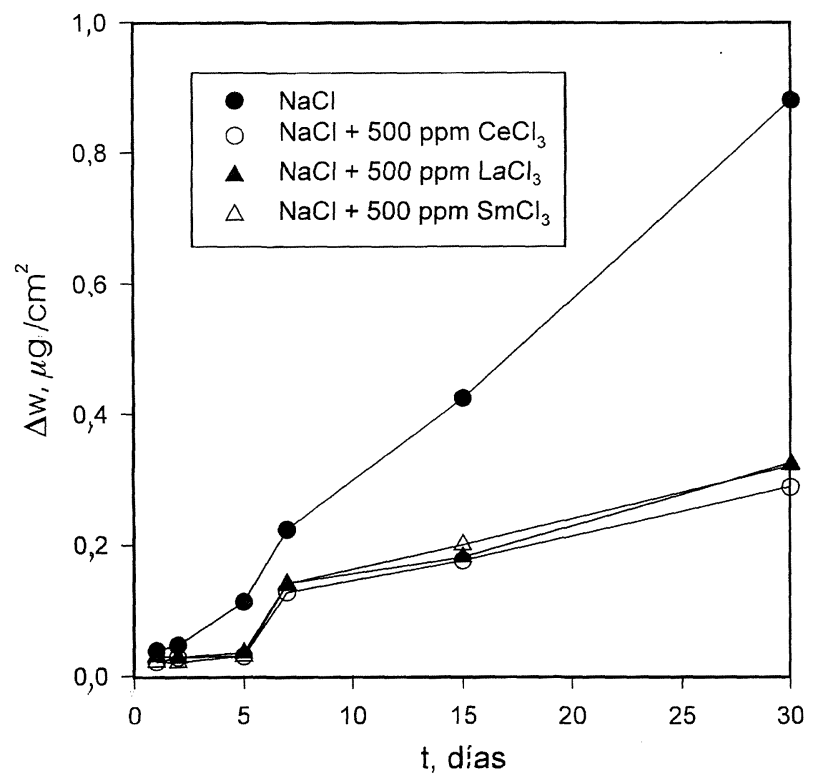

Fig. 1.- Curvas de pérdida de peso en función del tiempo de inmersión para probetas de la aleación AA5083 en disolución al 3,5\% de $\mathrm{NaCl}$, y en la misma disolución tras la adición de 500 ppm de $\mathrm{LnCl}_{3}$. Se incluye, como referencia, la curva correspondiente a ensayos realizados en disoluciones sin inhibidor.

FIG. 1.- Weight loss vs time curves obtained from full-inmersion tested samples of AA5083 alloy in solutions dopped with $500 \mathrm{ppm}$ of $\mathrm{LnCl}_{3}$. As a reference, the curve corresponding to samples tested in the bare solution has also been included.

en el tiempo la diferencia entre las pérdidas de peso se va haciendo cada vez mayor. La figura 2 ilustra la evolución con el tiempo de exposición de la pérdida de peso por adición de $500 \mathrm{ppm} \mathrm{de} \mathrm{LaCl}_{3}$ a la disolución base para las tres aleaciones estudiadas. Se puede observar que el descenso de la pérdida de peso es mucho más acusado para las probetas de aleación de aluminio que para las de acero.

Por medio de microscopía electrónica de barrido (MEB), se ha podido estudiar el estado superficial de las probetas procedentes de ensayos de inmersión de 30 días una vez eliminados los productos de corrosión. La figura 3a) corresponde a una muestra de la aleación 5083 ensayada en $\mathrm{NaCl}$. Se observa que la superficie aparece cubierta de picaduras. Al añadir $500 \mathrm{ppm}$ de $\mathrm{CeCl}_{3}$ a la disolución, se produce una fuerte inhibición del proceso de corrosión por picaduras. En efecto, la superficie de la muestra aparece completamente libre de picaduras después de estos ensayos (Fig. 3b). Resultados semejantes se han obtenido con los dos aceros estudiados. Estos resultados ponen de manifiesto el efecto inhibidor frente a la corrosión por picaduras de las sales lantánidas utilizadas.

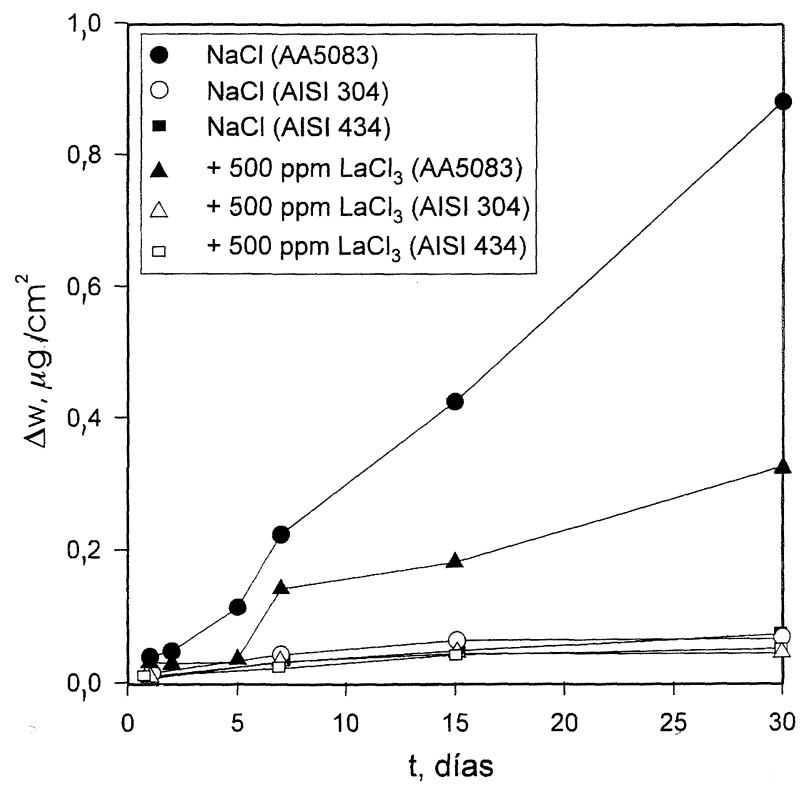

Fig. 2.- Curvas de pérdida de peso en función del tiempo de inmersión para las tres aleaciones estudiadas en disolución al 3,5\% de $\mathrm{NaCl}$, y en la misma disolución tras la adición de 500 ppm de $\mathrm{LaCl}_{3}$.

FIG. 2.- Weight loss vs time curves obtained from full immersion tested samples of the three alloys here studied in solutions dopped with 500 ppm of $\mathrm{LaCl}_{3}$.

Los ensayos de pérdida de peso no permiten evaluar separadamente las velocidades de corrosión uniforme y la resistencia a la corrosión por picaduras. Con objeto de poder realizar dicha evaluación, se efectuaron ensayos electroquímicos de polarización lineal y polarización cíclica. La figura 4 muestra los diagramas de polarización lineal para la aleación AA 5083, en disolución aireada de $\mathrm{NaCl}$ al 3,5 $\%$ en peso, con adición de cantidades comprendidas entre 100 y $1.000 \mathrm{ppm} \mathrm{de} \mathrm{CeCl}_{3}$. En la misma figura se incluye, a efectos comparativos, el diagrama de polarización lineal de la aleación en la disolución sin inhibidor.

Se puede observar que la presencia de la sal lantánida provoca un desplazamiento del diagrama de polarización en el sentido de disminuir el potencial de corrosión. Esto lleva asociado un descenso de la densidad de corriente de corrosión y, en consecuencia, de la velocidad de corrosión. El mayor desplazamiento se obtiene para una cantidad de $500 \mathrm{ppm}$ de sal lantánida. Se aprecia cómo la cantidad de $1.000 \mathrm{ppm}$ de inhibidor provoca un desplazamiento, menos acusado que para cantidades inferiores, asociable al aumento de la concentración de iones cloruro en la disolución (15). De acuerdo con (1), las modificaciones del diagrama de polarización lineal producidas por la presencia de las sales $\mathrm{LnCl}_{3}$, ponen de manifiesto el carácter catódico del 


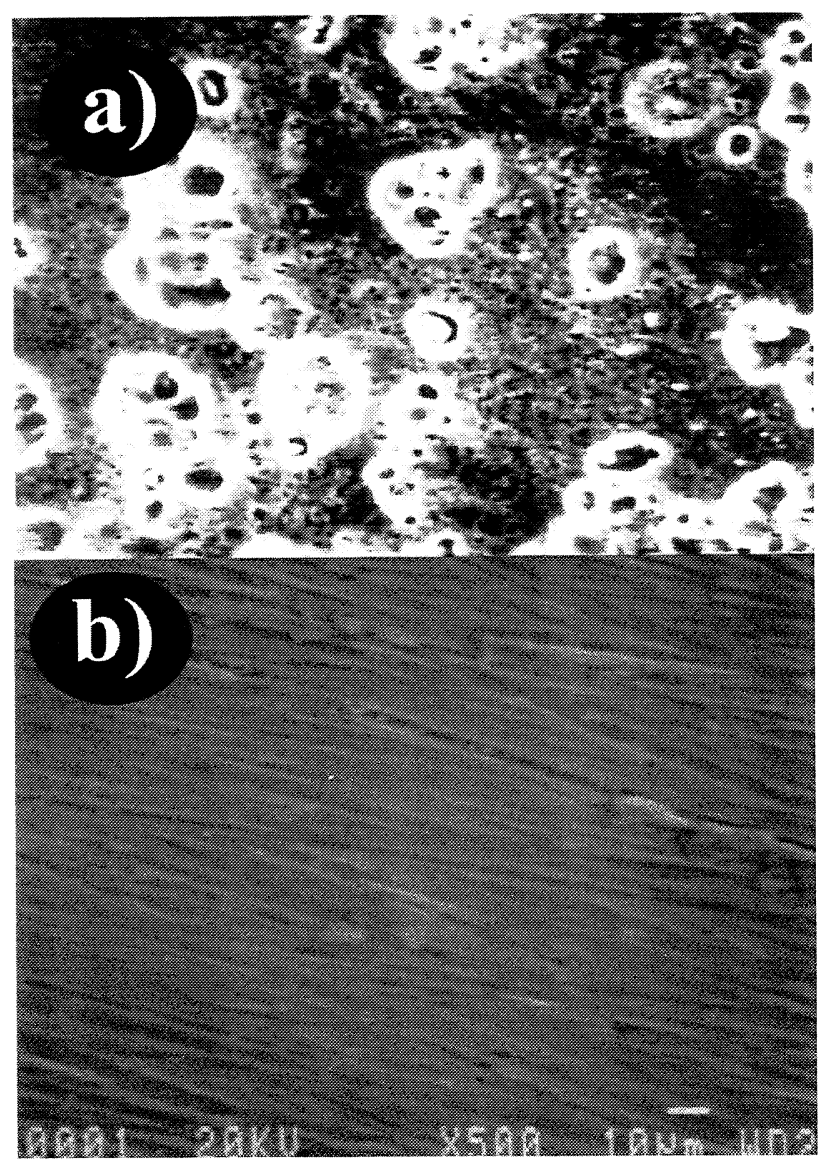

FIG. 3.- Imágenes obtenidas con MEB correspondientes a probetas de la aleación AA5083 tras 30 días de inmersión en disolución aireada de $\mathrm{NaCl}$ al $3,5 \%$ : a) sin inhibidor, b) con adición de 500 ppm de $\mathrm{CeCl}_{3}$.

FIG. 3.- SEM images corresponding to an AA5083 alloy sample after 30 days of exposure to an aerated $3.5 \% \mathrm{NaCl}$ solution: a) without inhibitor, and b) with $500 \mathrm{ppm}$ of $\mathrm{CeCl}_{3}$.

proceso de inhibición. Según Hinton (15-17) este proceso puede tener lugar por precipitación de un "hidróxido" lantánido en las zonas catódicas del material.

Los análisis con MEB y con EDS han servido para obtener datos que confirman la existencia de un mecanismo de actuación semejante sobre las aleaciones aquí estudiadas. En las figuras $5 \mathrm{a}$ ) y $5 \mathrm{~b}$ ) se muestran, respectivamente, las imágenes obtenidas con MEB de muestras de acero AISI 434 y de la aleación AA 5083 después de someterlas a ensayos de inmersión en disoluciones aireadas de $\mathrm{NaCl}$ al $3,5 \%$ con $500 \mathrm{ppm}$ de $\mathrm{CeCl}_{3}$ y de $\mathrm{LaCl}_{3}$. En dicha figura se observa cómo durante los ensayos se forma una película delgada y bien adherida sobre la superficie de los materiales estudiados. Las representaciones espectrales de esta figura muestran picos característicos de lantano (Fig. 5c) y de cerio (Fig. 5d). Estas capas, según se ha comentado anteriormente, se formarían por precipitación del catión

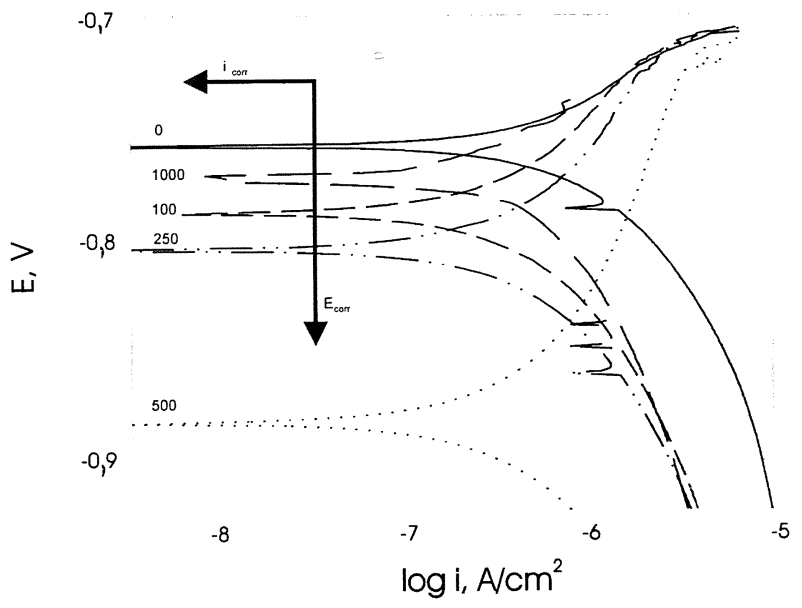

FIG. 4.- Curvas de polarización lineal de la aleación AA5083 en disoluciones aireadas de $\mathrm{NaCl}$ al $3,5 \%$, conteniendo cantidades variables de $\mathrm{CeCl}_{3}$.

FIG. 4.- Linear Polarisation diagrams of the AA5083 alloy in aerated $3.5 \% \mathrm{NaCl}$ solutions with $\mathrm{CeCl}_{3}$ additions ranging from 0 to 1,000 ppm.

lantánido con los grupos hidroxilos generados en la semirreacción catódica. De acuerdo con los resultados expuestos, la formación de estas capas es la responsable del efecto inhibidor observado.

La protección que se consigue con las sales lantánidas se puede evaluar en términos del porcentaje de reducción de la velocidad de corrosión uniforme calculada a partir de los diagramas de polarización lineal. En la figura 6 se representa la variación del grado de protección en función de la concentración de inhibidor $\left(\mathrm{LaCl}_{3}\right)$. Se observa que para los tres materiales ensayados los valores porcentuales máximos de protección se obtienen para una concentración de inhibidor de 500 ppm; se alcanzan valores próximos al $70 \%$ para los aceros y superiores al $80 \%$ para la aleación de aluminio. Estos valores porcentuales se mantienen en el mismo orden que los aportados por los cromatos, por lo que estas sustancias se pueden considerar como alternativas efectivas al uso de esos compuestos.

Para evaluar la inhibición de la corrosión por picaduras se efectuaron ensayos de polarización cíclica (Fig. 7). El diagrama correspondiente a la aleación AA 5083 (Fig. 7b), presenta un ciclo de histéresis anódica completo. En el caso de los aceros inoxidables (Fig. 7a), el ciclo se cerraría en la rama catódica, lo que significa que no existe repasivación o, lo que es lo mismo, que una vez formadas las picaduras crecerían indefinidamente. Este comportamiento de los aceros se asocia a la saturación de oxígeno del medio (26). Como se puede observar en la figura 7, en ambos casos, al añadir la sal lantánida se produce un desplazamiento del 

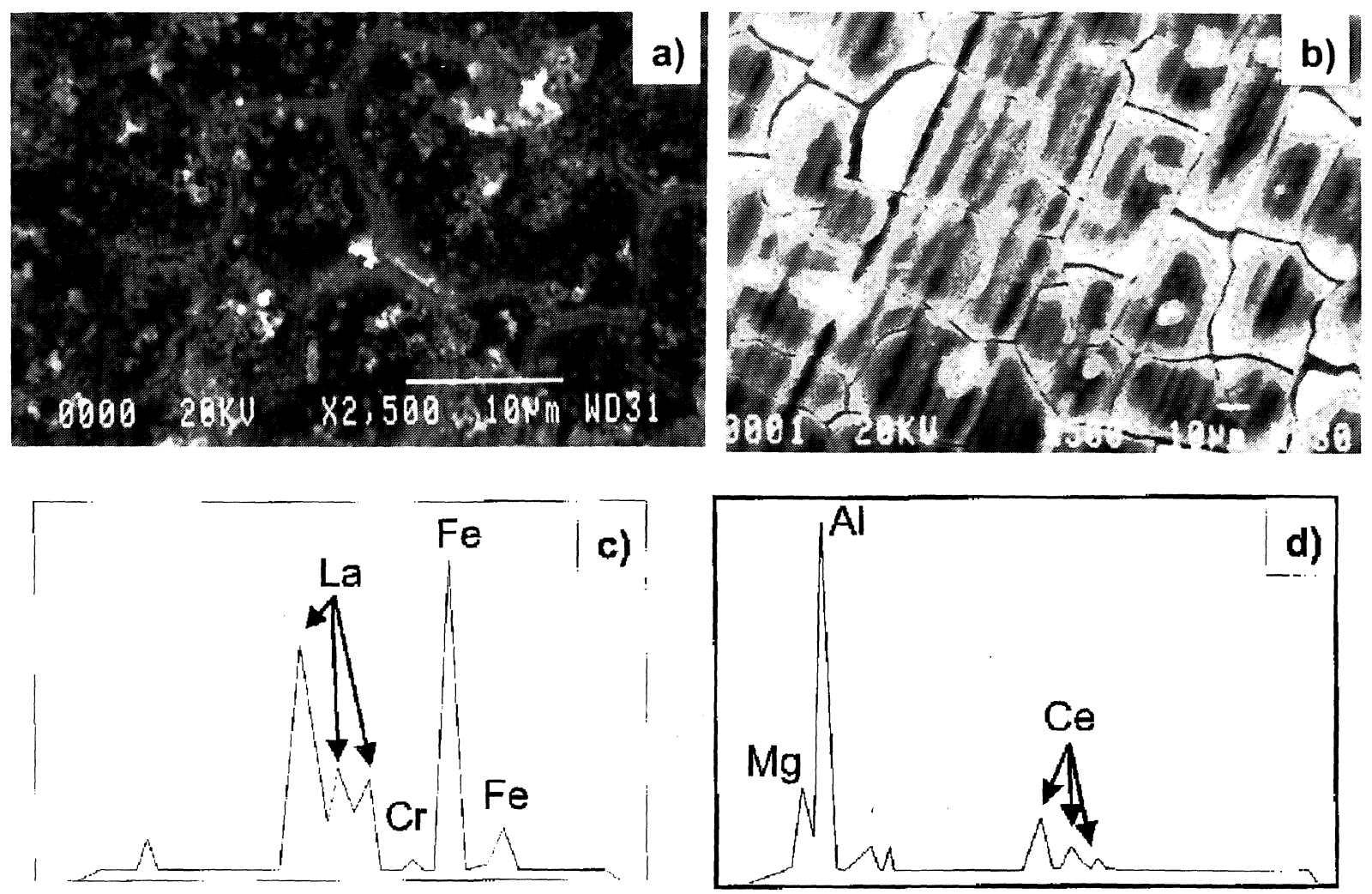

FIG. 5.- Imágenes obtenidas con MEB correspondientes a probetas de: a) AISI 434, y b) AA5083, tras 30 días de ensayo en la disolución $\mathrm{NaCl}$ con $500 \mathrm{ppm}$ de $\mathrm{LaCl}_{3}$ y $\mathrm{CeCl}_{3}$, respectivamente. En ambos casos, se observa la presencia de una película sobre la superficie del metal. c) y d) Las gráficas

espectrales obtenidas con EDS evidencian la incorporación de los elementos lantánidos a las capas.

FIG. 5.- SEM images of: a) AISI 434, an b) AA5083 after a 30 days full immersion test in a $\mathrm{NaCl}$ solution with 500 ppm of $\mathrm{LaCl}_{3}$ and $\mathrm{CeCl}_{3}$ respectively. Notice the film formed onto the two metallic surfaces. c) and d) The EDS spectra show the incorporation of the lanthanide elements into the films.

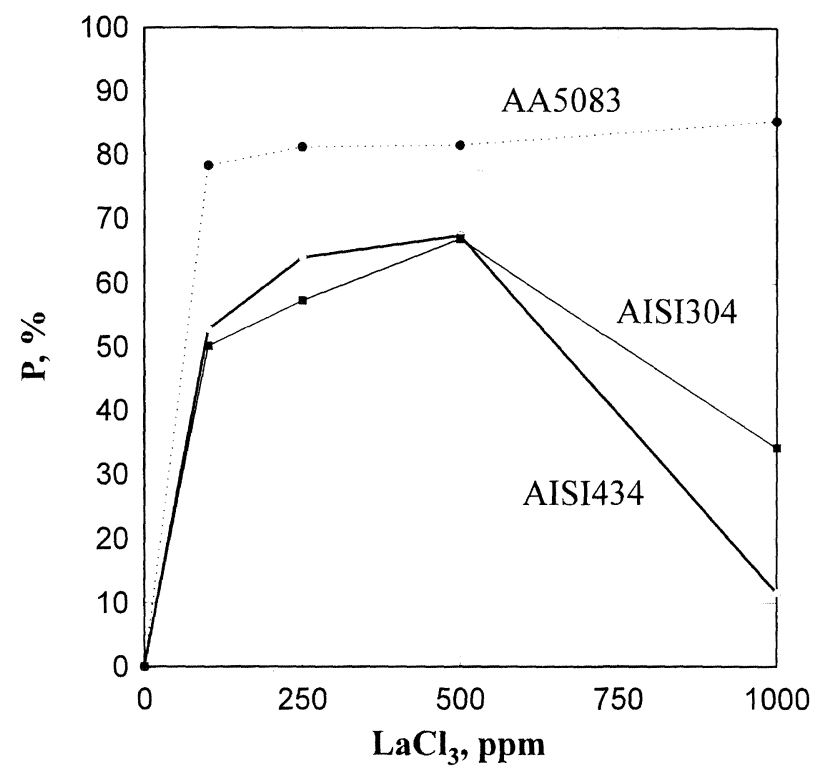

FIG. 6.- Porcentaje de reducción de $v_{\text {corr }}$ en función de la concentración de $\mathrm{LaCl}_{3}$ para los tres materiales ensayados.

FIG. 6.- Porcentage of decrease in $\mathrm{v}_{\text {corr }}$ vs $\mathrm{LaCl}_{3}$ concentration for the three studied alloys. potencial de corrosión hacia valores más activos, mientras que apenas existe diferencia en el potencial de nucleación de picaduras. Una medida de la resistencia a la formación de picaduras, $R_{\mathrm{np}}$, viene dada por la diferencia entre los potenciales de nucleación de picaduras, $E_{\mathrm{np}}$, y de corrosión, $E_{\text {corr }}$ (27). Por tanto, de acuerdo con los datos incluidos en la figura 7, al añadir la sal lantánida se obtiene un aumento de la resistencia a la formación de picaduras. La protección que se consigue con la adición de inhibidor se puede evaluar en términos del porcentaje de aumento de $R_{\mathrm{np}}$. En la figura 8 se ilustra la evolución de este parámetro en función de la concentración de $\mathrm{CeCl}_{3}$ para cada aleación estudiada. Como se puede observar, la máxima protección conseguida frente a la formación de picaduras se alcanza al añadir al medio $500 \mathrm{ppm}$ de $\mathrm{CeCl}_{3}$.

Además de evaluar la protección frente a la formación de picaduras, haciendo uso del método descrito en (24) se ha evaluado la protección conseguida con los inhibidores contra el crecimiento de picaduras preexistentes. Este método está basado en la comparación de las áreas de los ciclos de histéresis anódica. Dicha área se puede asociar con la 

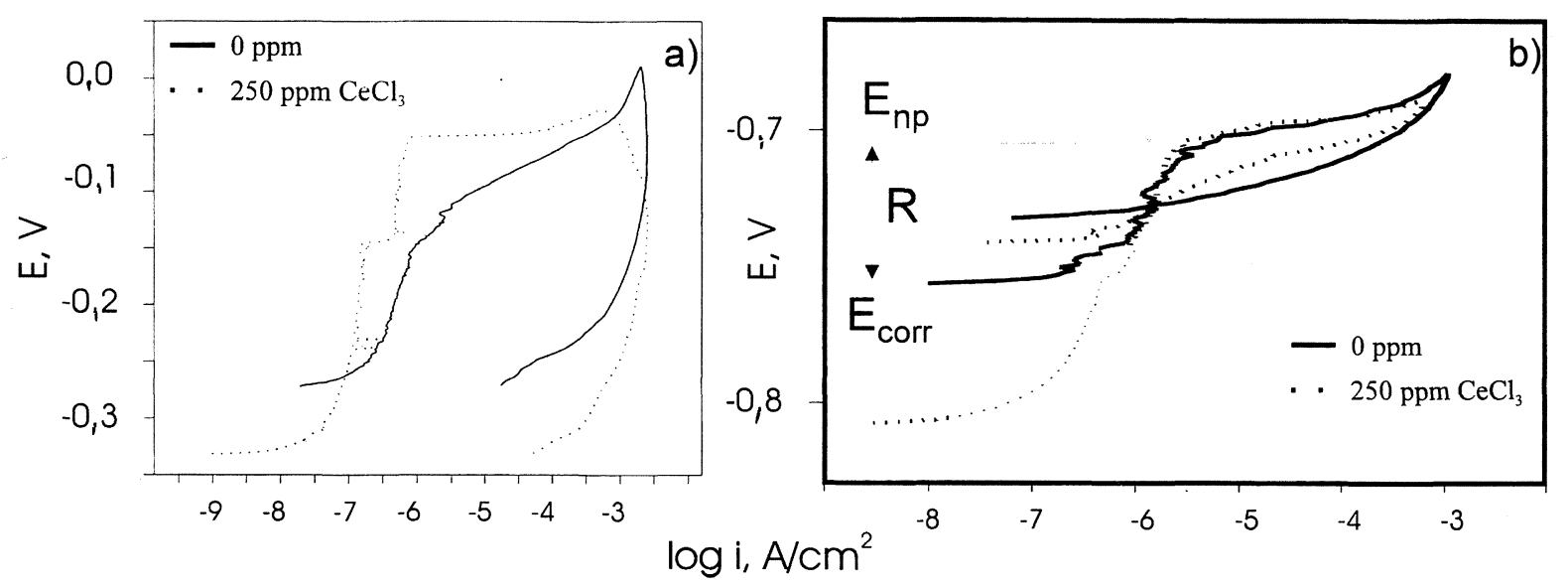

FIG. 7.- Curvas de polarización cíclica correspondientes a los materiales: a) AISI 434, y b) AA5083, en disoluciones aireadas de $\mathrm{NaCl}$ al 3,5\%.

FIG. 7.-Cyclic polarisation diagrams of: a) AISI 434, an b) AA5083 alloys in aerated NaCl solutions. Notice the effect of the addition of $\mathrm{CeCl}_{3}$.

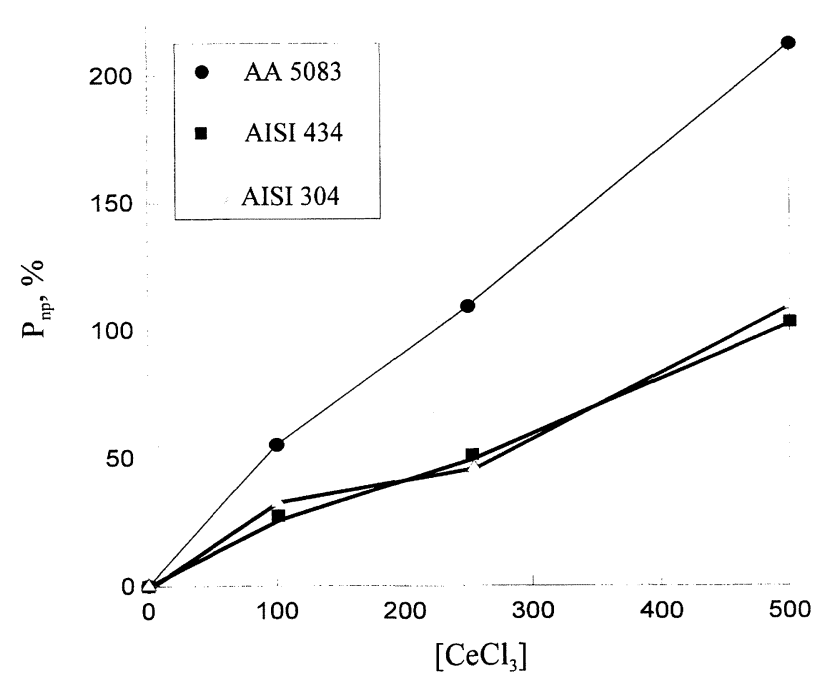

FIG. 8.- Resistencia a la nucleación de picaduras en función de la concentración de $\mathrm{CeCl}_{3}$ añadido a la disolución, para los tres materiales estudiados.

FIG. 8.- Pits nucleation resistance as a function of $\mathrm{CeCl}_{3}$ concentration for the three studied materials.

potencia consumida durante el proceso de crecimiento de las picaduras, previamente formadas al alcanzarse el potencial de ruptura. Según se desprende de la figura 7, en el presente caso este método sólo es aplicable a la aleación de aluminio. Para la aleación AA 5083, el grado de protección frente al crecimiento de las picaduras se ha evaluado en términos del porcentaje de reducción del área del ciclo. La figura 9 muestra la evolución de este valor porcentual en función de la concentración de cada inhibidor empleado. Se deduce que el mayor efecto protector se obtiene para una concentración de 500 ppm de $\mathrm{CeCl}_{3}$ o de $\mathrm{LaCl}_{3}$.

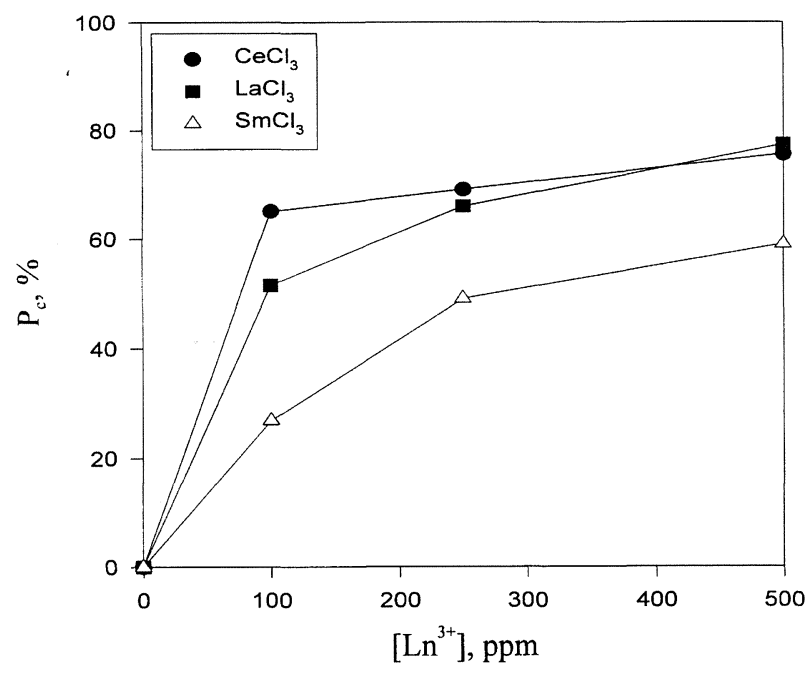

FIG. 9.- Porcentaje de protección frente al crecimiento de picaduras en la aleación AA5083, en función de la concentración de $\mathrm{LnCl}_{3}$ en la disolución.

FIG. 9.- Porcentage of protection against pitting growth in AA5083 alloy as a function of $\mathrm{LnCl}_{3}$ contents in solution.

\section{CONCLUSIONES}

De los datos obtenidos de los ensayos de pérdida de peso, se concluye que la adición de $\mathrm{LaCl}_{3}, \mathrm{CeCl}_{3}$ y $\mathrm{SmCl}_{3}$ inhibe el proceso de corrosión uniforme de los aceros AISI 434 y 304, así como el de la aleación de aluminio y magnesio AA 5083 en disoluciones aireadas de $\mathrm{NaCl}$ al 3,5\% en peso a temperatura ambiente. Además, la ausencia de picaduras en las probetas sumergidas en las disoluciones que contienen $\mathrm{LnCl}_{3}$, permite deducir que estas sales 
actúan también como inhibidoras de la corrosión por picaduras.

Las curvas de polarización lineal sugieren que el proceso de inhibición tiene un carácter catódico. En este sentido, y de acuerdo con (10, 16, 22 y 24), el proceso de inhibición tendría lugar por la precipitación de un óxido o hidróxido lantánido en las zonas catódicas de la superficie metálica, formando finalmente una película protectora como la que revelan los ensayos con MEB y EDS. Esta película actúa como una barrera protectora entre el metal y el medio agresivo, bloqueando la reacción catódica y, por lo tanto, disminuyendo la velocidad de corrosión.

Los datos obtenidos con los ensayos de polarización cíclica han permitido determinar el grado de protección frente a la nucleación de picaduras para los tres tipos de aleación estudiados. Los porcentajes de protección contra la nucleación de picaduras han llegado a superar el $200 \%$. Por otro lado, el crecimiento de las picaduras ya formadas en la aleación AA 5083 se llega a reducir en un $70 \%$. En ambos casos, la concentración óptima de inhibidor se sitúa en torno a las 500 ppm.

En resumen, los cloruros lantánidos investigados se comportan de forma eficaz en la inhibición de los procesos de corrosión uniforme y por picaduras de los tres materiales estudiados. Los factores de protección obtenidos son del mismo orden que los encontrados para los inhibidores basados en compuestos de Cr(VI). Este hecho, unido a la baja toxicidad de las sales lantánidas, permite considerar a éstas como una alternativa ecológica eficiente al empleo de los cromatos.

\section{Agradecimiento}

Este trabajo ha sido financiado por la CICYT, Proyecto MAT 95-2011, y por la Junta de Andalucía, Proyecto PAI-CA3.

\section{REFERENCIAS}

(1) B.G. Clubey (Ed.). Chemical Inhibitors for Corrosion Control. The Royal Society of Chemistry. 1990.

(2) Bahadur, A. Corr. Rev., 10 (1-2), 1992: 155.

(3) U.S. Public Health Service. Report No. ASTDR/TP88/10, 1989.
(4) Garton, R. Biological Effects of Cooling Tower Blowdown. NERC. Corvallis, 1987.

(5) CoHen, S.M. Corrosion, 51 (5), 1995: 71.

(6) Chang, T.C. Report No. NMDC-K5784. McDonell Douglas Corp. 1991.

(7) Hedenquist, P. y Roos, A. Surf. Coating Tech., 48, 1991: 41.

(8) Brown, S.D. y WirTZ, G.P. Proc. Symp. on Al Surf. Treatment Tech. 11. Eds. R.S. Alwitt y G.E. Thompson. New Jersey (EE.UU.), 1986: 138.

(9) Kato, K. J. Mater. Sci., 28, 1993: 4.033.

(10) Aldykewicz, A.J., ISAacs, H.S. y Davenport, A.J. $J$. Electrochem. Soc., 143 (1), 1996: 147.

(11) Aldykewicz, A.J., Isaacs, H.S. y Davenport, A.J. $J$. Electrochem. Soc., 142 (10), 1995: 3.342.

(12) IsAacs, H.S. y DAVENPORT, A.J. J. Electrochem. Soc., 138 (2), 1991: 390.

(13) Mansfeld, F. y Wang, Y. Corrosion'95, NACE, Paper No. 95041. Orlando (EE.UU.), 1995.

(14) Mansfeld, F., Wang, V. y Shin, H. J. Electrochem. Soc., 138 (12), 1991: L74.

(15) Hinton, B.R.W., Ryan, N.E. y ArnotT, D.R. Mat. Australasia, Jan.-Feb., 1987: 42.

(16) Arnott, D.R., Hinton, B.R.W. y Ryan, N.E. Mat. Perf., Aug. 1987: 42.

(17) Hinton, B.R.W., Ryan, N.E. y Arnott, D.R. Mat. Forum, 9 (3), 1986: 162.

(18) Kendig, M. y Thomas, C. J. Electrochem. Soc., 139 (11), 1992: L103.

(19) Bernal, S., Botana, F.J., Calvino, J.J., Cauqui, M.A., Marcos, M., Pérez, J.A. y Vidal, H. Proc. 13th Int. Cong. on Elect. Microscopy. París (Francia), 1994: 1.105.

(20) Bernal, S., Botana, F.J., Calvino, J.J., Cauqui, M.A., Marcos, M., Pérez, J.A. y Vidal, H. Proc. 2nd Int. Conf. on f-Elements. Helsinkii (Finlandia), 1994: 354.

(21) Bernal, S., Botana, F.J., Calvino, J.J., Cauqui, M.A., Marcos, M., Pérez, J.A. y Vidal, H. Proc. 5th Electrochemical Methods in Corrosion Research. Sesimbra (Portugal), 1994: P I1.

(22) Bernal, S., Botana, F.J., Calvino, J.J., Cauqui, M.A., Marcos, M., Pérez, J.A. y Vidal, H. J. Alloys Comp., 225, 1995: 638 .

(23) Bethencourt, M., Botana, F.J., Calvino, J.J., Cauqui, M.A., Marcos, M. y Rodriguez, M.A. Proc. XI Cong. Iberoam. de Corr. y Protección. Tenerife, 1995: 321.

(24) Bethencourt, M., Botana, F.J., Cauqui, M.A., Marcos, M., Rodriguez, M.A. y Rodriguez-IzQuierdo, J.M. J. Alloys Comp. (En prensa).

(25) Registry of Toxic Effects of Chemical Substances, 86, DHHS-NIOSH Publication. Nueva York (EE.UU.), 1986: 103.

(26) Lu, Y.C. e Ives, M.B. Corr. Sci., 34 (11), 1993: 1.173.

(27) SzKLARSKa-SmialowsKa, Z. Pitting Corrosion of Metals. NACE, Houston (EE.UU.), 1986. 